\title{
Shot Boundary Detection Using Frame Transition Parameters and Edge Strength Scatter
}

\author{
P.P. Mohanta ${ }^{1}$, S.K. Saha ${ }^{2}$, and B. Chanda ${ }^{1}$ \\ ${ }^{1}$ ECS Unit, Indian Statistical Institute, Kolkata, India \\ ${ }^{2}$ CSE Department, Jadavpur University, Kolkata, India
}

\begin{abstract}
We have presented a unified model for various types of video shot transitions. Based on that model, we adhere to frame estimation scheme using previous and next frames. The frame parameters accompanied by a scatter measure of edge strength and average intensity constitute the feature vector of a frame. Finally, the frames are classified as no change (within shot frame), abrupt change or gradual change frames using a multilayer perceptron network. The scheme is free from the problems of selecting thresholds and/or window size as used by various schemes. Moreover, the handling of both, abrupt and gradual transitions along with non-transition frames under a single and uniform framework is the unique feature of the work.
\end{abstract}

Keywords: shot detection, abrupt transitions, cut, gradual transitions.

\section{Introduction}

Due to the advancement of video technology the volume of digital video data has increased dramatically. But, the tools available for browsing such databases are still primitive in nature. To address the problem, indexing and retrieval has become an active area of research. Video segmentation is the fundamental step for the said application including video indexing, content analysis of video sequence, video accessing, retrieving and browsing, video compression and others. A fast and automatic technique for temporal segmentation of video content is very crucial for accurate content description.

The objective of video shot segmentation is to partition video into meaningful and basic structural units called shots. A shot corresponds to a sequence of frames captured through a continuous record (in time and space) by camera [1]. It describes a meaningful event over a continuous sequence of frames. Once the boundaries of the shots are detected, further analysis of content and interpretation can be performed on such units.

The transition may be of various types and broadly categorized as abrupt and gradual transition. Abrupt transition is also known as cut and it denotes instantaneous transition from one shot to another. On the other hand, a gradual transition is obtained by incorporating photographic effect usually through editing. It can be further classified as fade-in, fade-out and dissolve. Fade-out is 
a gradual transition of a scene to a constant image (commonly a black frame) and fade-in is reverse transition. Dissolve is gradual super-imposition of two consecutive shot.

It is easy to detect the cuts as it involves two successive frames which are highly uncorrelated and differs significantly. But, during gradual transition, the successive frames may not differ much. Thus, the major challenge becomes to distinguish between the gradual transition and the nominal changes in the scene.

Lots of work has been reported on cut detection. Comparatively, a less amount of work has dealt with gradual transition. The basis of all such algorithms lies in detecting the visual discontinuities in time domain. The schemes extract the visual features and deploys a similarity between the frames. Most of the cut detection schemes identifies the transition if the difference between two consecutive frames exceeds a certain threshold. Similarity (difference) of the frames are measured in terms of features computed from the frame. A wide variety of features have been reported in various works. The simplest one is pixel wise difference 2[3]. But as it is very sensitive to motion of objects, grayscale/colour histogram based features are also tried in 4] though the histograms lack spatial information. As an alternative, features based on motion vector analysis [5], edge tracking [6, edge changes [7, entropy measures [8] are also used. In order to detect gradual transitions, twin comparison method [2] deals with two threshold values to detect cuts and gradual transition. Yeo and Lin [3] proposed plateau detection technique where difference between current frame and $k$ th frame that follows is considered. But, in this case, proper selection of $k$ is a non-trivial task. A method called chromatic scaling has been discussed in [9]. In another approach, transitions are detected by counting the entering/exiting edge pixels [10. Machine learning and multi resolution concept 11] are also reported for dissolve identification. Algorithm evolved by combining the concept of object tracking and feature based approaches was also tried for dissolve detection [12]. In 13, the variance of pixel intensity of a sequence is modeled as a parabolic curve and based on that model a detection scheme is presented.

It appears that although a lot of schemes have been tried but they have their own merits and demerits and almost none of them has tried to detect all kinds of shot boundaries in a comprehensive way. In this work, we present a parametric model of the shot transitions of various types which, in turn, will be used to detect and classify the shot. The paper is organized as follows. Section 2 presents the formulation of the problem and the details of the proposed scheme. Experimental results are presented in section 3 and concluding remarks are put in section 4 .

\section{Proposed Methodology}

In this section, a general framework is presented to describe the transition of various types and it will act as the basis for the proposed shot detection scheme.

In case of abrupt transition, last frame of a shot and first one of the following shot are uncorrelated. A cut is generated by the natural process of capturing 
video data through the camera. On the contrary, gradual transitions (fade-in, fade-out and dissolve or cross-fading) are generated through editing. Dissolves are generated by super-imposing the boundary frames of two successive shots over a duration. In such cases of gradual transition, intensity of one boundary frame gradually decreases and that of other one increases during the phase of transition. For fade-out (or fade-in) the intensity of boundary frames are gradually reduced (or increased) and last (or first) frame of such transitions is commonly a black frame. Thus, unlike abrupt transitions, gradual transitions spans over a range of frames. It is also obvious that presence of motion and activities usually are very insignificant in the frames of such edited transitions.

The successive frames within a shot and also those within the span of a gradual transition show little differences. Thus, the ability to distinguish the two situation controls the false and misclassification rate. It may be noted that, differences between the successive frames within a shot is mostly caused by camera and/or object motion keeping the background otherwise unaltered. But, for gradual transition, it mainly comes from the editing process.

\subsection{Problem Formulation}

With the background idea of natural and edited transitions, we formulate the scenario as follows.

Let, $f_{1}, f_{2}, \ldots, f_{n}$ denotes a sequence of frames in a video. Suppose, $f_{l_{1}}$ and $f_{l_{2}}$ are the last representative frame of a shot and the first representative frame of the following shot, where, $0 \leq l_{1}<l_{2} \leq n$. The frames in transitions are denoted by $f_{i}$ where $i$ varies from $l_{1}$ to $l_{2}$. Such frames may be represented as

$$
f_{i}=A_{i} f_{l_{1}}+B_{i} f_{l_{2}}
$$

where, $0 \leq A_{i}, B_{i} \leq 1$ and $A_{i}+B_{i}=1$. Basically, $A_{i}$ 's and $B_{i}$ 's modulates the intensities of the frames being super-imposed.

In case of a cut, $l_{2}=l_{1}+1$. From equation [1, it is obvious that there will be two transition frames with $A_{i}=1, B_{i}=0$ for one and reverse for the other.

For gradual transition, $A_{i}$ gradually decreases from 1 to 0 and $B_{i}$ successively increases from 0 to 1 . It may be noted that, in case of fade-in, $f_{l_{1}}$ is the black frame and for fade-out, $f_{l_{2}}$ is the black frame.

Thus, the model shown in equation 1 can represent all sorts of transitions. But, during the process of boundary detection, the representative frames $\left(f_{l_{1}}\right.$ and $f_{l_{2}}$ ) are not available. As a matter of fact, it is the task of detection process to find out these frames along with the boundaries. Even then, model described in equation 1 provides the underlying structure for our scheme. Based on the model of equation 1, $f_{i-1}, f_{i}$ and $f_{i+1}$ can be represented as follows.

$$
\begin{gathered}
f_{i-1}=A_{i-1} f_{l_{1}}+B_{i-1} f_{l_{2}} \\
f_{i}=A_{i} f_{l_{1}}+B_{i} f_{l_{2}}
\end{gathered}
$$




$$
f_{i+1}=A_{i+1} f_{l_{1}}+B_{i+1} f_{l_{2}}
$$

By manipulating the equations 2, 3 and 4 $f_{i}$ can be represented in terms of its previous and following frame as shown in equation 5 .

$$
f_{i}=a_{i} f_{i-1}+b_{i} f_{i+1}
$$

where $a_{i}=\frac{A_{i} B_{i+1}-B_{i} A_{i+1}}{A_{i-1} B_{i+1}-A_{i+1} B_{i-1}}$ and $b_{i}=\frac{B_{i} A_{i-1}-A_{i} B_{i-1}}{A_{i-1} B_{i+1}-A_{i+1} B_{i-1}}$, and $A_{i}+B_{i}=1$ implies that $a_{i}+b_{i}=1$. This model is also valid for the frames within a shot (i.e. no change frames) and in that case, ideally, it will be $a_{i}=b_{i}=0.5$. Thus, all types of frames can be estimated from its previous and following frame. Ideally, the characteristic pattern of $\left(a_{i}, b_{i}\right)$ is similar to that of $\left(A_{i}, B_{i}\right)$ and can be used for shot detection and classification purpose. This has motivated us to go for frame parameter estimation for shot boundary detection and classification. As we adhere to the model of equation 5 instead of that in equation 1, there is no need to consider a sliding window of suitable size for studying the characteristics of $\left(a_{i}, b_{i}\right)$. Thus, our methodology will remain free from the burden of selecting the window size as it deals with only previous and next frame.

\subsection{Computation of Frame Transition Parameters}

A frame (or image) in a video sequence consists of two major types of components: background and foreground objects. Over the frames background is either static (no change) or may undergo little motion due to camera pan and tilt. On the other hand, foreground objects exhibit activities including significant motion. To incorporate both types of characteristics in the frame transition parameter estimation we use both global and local (edge scatter) features.

Estimation based on global feature: Let $f_{i_{e}}$ denotes the estimate for $i$-th frame using the equation 5 with appropriate parameters $\left(a_{i}, b_{i}\right)$. As mentioned earlier, a frame consists of background and foreground or active objects. Consistency of the background can better be represented in terms of global features. Since we have to deal with a huge amount of data, it is advisable to use some features which need as little computation as possible. In the proposed method we have used gray level histogram of the frames.

For the time being, let us consider that the frames are continuous domain containing continuous value of intensity. Thus gray level histogram may be treated as probability density function (p.d.f.) and let the p.d.f. of frame $f_{i}$ is denoted by $p_{i}\left(v_{i}\right)$, where $v_{i}=f_{i}(x, y)$; so that the Jacobian of the linear transformation may be applied to estimate the p.d.f. of the candidate frame from the p.d.f's of the subsequent and the previous frame. Equation 5 suggests that the intensity of $i$-th frame is obtained by linear transformation of the $(i-1)$-th and $(i+1)$-th frames. Thus, the p.d.f. of frame $f_{i}$ may be obtained from $p_{i-1}\left(v_{i-1}\right)$ and $p_{i+1}\left(v_{i+1}\right)$. To make the formulation mathematically tractable and computationally efficient we assume that $v_{i-1}$ and $v_{i+1}$ are independently distributed. Thus, the joint distribution of $v_{i-1}$ and $v_{i+1}$ is defined as $p\left(v_{i-1}, v_{i+1}\right)=p_{i-1}\left(v_{i-1}\right) \times p_{i+1}\left(v_{i+1}\right)$. To derive the distribution of $v_{i_{e}}$ let 
$v_{i_{e}}=a_{i} v_{i-1}+b_{i} v_{i+1}$ and $u=v_{i+1}$ which implies $v_{i-1}=\frac{v_{i_{e}}-b_{i} u}{a_{i}}$. Now, the Jacobian of the transformation is $|J|=\left|\frac{\delta\left(v_{i-1}, v_{i+1}\right)}{\delta\left(v_{i_{e}}, u\right)}\right|=\frac{1}{a_{i}}$

Thus, the joint distribution of $v_{i_{e}}$ and $u$ is $p\left(v_{i_{e}}, u\right)=p_{i-1}\left(\frac{v_{i_{e}}-b_{i} u}{a_{i}}\right) \times p_{i+1}(u) \times \frac{1}{a_{i}}$ The distribution of $v_{i_{e}}$ is

$$
p_{i_{e}}\left(v_{i_{e}}\right)=\frac{1}{a_{i}} \int p_{i-1}\left(\frac{v_{i_{e}}-b_{i} u}{a_{i}}\right) p_{i+1}(u) d u
$$

Hence, by minimizing the error between the actual p.d.f. $p_{i}\left(v_{i}\right)$ and the estimated p.d.f. $p_{i_{e}}\left(v_{i_{e}}\right)$ we obtain the appropriate values of parameters $\left(a_{i}, b_{i}\right)$ which characterize the frame transition. In this work the said error is measured as the Bhattacharya distance.

Actually, the estimation process is carried out based on the intensity histogram of the frames. Thus, $p_{i_{e}}, p_{i}, p_{i-1}$ and $p_{i+1}$ represent the intensity histogram of respective frames. We try to find out the values of $a_{i}$ and $b_{i}$ to obtain the best estimate for $p_{i}$. Along with those, $E_{i}$, the error of estimation is also taken as a feature of estimate. The computation steps are as follows.

- $p_{i-1}, p_{i}$ and $p_{i+1}$, the normalized 256 bin intensity histograms are computed.

$-p_{i-1}$ and $p_{i+1}$ are shifted to make $\mu_{i}=\mu_{i-1}=\mu_{i+1}$, where $\mu_{i}$ is the average intensity value of the $i$-th frame.

- Exhaustive search for $a_{i}, b_{i}$ is employed to attain optimum $E_{i}$.

- $E_{i}=\operatorname{dist}\left(p_{i_{e}}, p_{i}\right)$, the error between the frames $f_{i_{e}}$ and $f_{i}$.

- $a_{i}, b_{i}$ and $E_{i}$, the estimated error for the best $\left(a_{i}, b_{i}\right)$, are taken as transition characteristic features for the $i$-th frame.

It may be noted that in case of cut $f_{i}$ and $f_{i-1}$ or $f_{i}$ and $f_{i+1}$ are highly uncorrelated suggested by either $a_{i}$ or $b_{i}$ equal to zero. On the other hand, $f_{i-1}$, $f_{i}$ and $f_{i+1}$ are strongly correlated in case of no change and gradual transition. This is revealed by non-zero values of both $a_{i}$ and $b_{i}$ in estimating these frames. This may lead to confusion in identifying within shot frames and dissolve frames. However, it may be shown that the within shot frames can be estimated from either previous or next frame only, which is not possible in case of dissolve frames. This suggests a strong distinction between them. So, in order to reduce the conflict in detection and classification, we further consider the estimation process using only the previous frame and only the next frame. The equations for such estimation are as follows.

$$
\begin{aligned}
& f_{i_{e}}=a_{i_{p}} f_{i-1} \\
& f_{i_{e}}=b_{i_{n}} f_{i+1}
\end{aligned}
$$

As in earlier case, the parameters $a_{i_{p}}, b_{i_{n}}$ and error of estimation $e_{p}, e_{n}$ are used as features. Average intensity $\left(I_{a v g}\right)$ of the frame is also taken as a parameter. In identification of fade-in and fade-out, $I_{a v g}$ contributes significantly. For fade-in, $I_{a v g}$ will show a gradual increase and it is reverse for fade-out. 
Estimation based on local features: Object motion as well as the gradual shift (if any) of the background may be arrested by detecting shift of edge points over the frames. This information may be extracted using neighborhood operators and are treated as local features. Edge strength (gradient magnitude at edge points) between two successive frames change due to change of $a_{i}$ and $b_{i}$ of equation 5 or motion or both. Thus scatter matrix of edge strength of two successive frames provides a representation of intensity transition as well as motion. It may be noted that if there is no motion, the scatter matrix for two successive within shot frames is, ideally, a diagonal matrix and it is a banded diagonal in case of gradual transition only. In case of abrupt transition the scatter matrix deviates significantly from the diagonal form. Non-diagonal elements would be loaded even more if motion is included. However, edge strength of within shot frames or gradual change frames, where motion is small and regular, is usually aligned or accumulated along the diagonal of the scatter matrix if the shifted position of the edge points can be found. This calls for solving the well known correspondence problem. If motion shifts a point at most by $K$ in any direction and object width is more than $2 K$, then we simply solve the correspondence problem by taking two similar edge points (in terms of relative magnitude and direction) of two frames within $K \times K$ window as the original and the shifted edge point.

Implementation of Scatter matrix and computation of local feature $S_{m}$ as follows. Let, $g_{i-1}, g_{i}$ and $g_{i+1}$ denote the gradient images corresponding to $(i-1)$-th, $i$ th and $(i+1)$-th frame. The gradient images are subjected to a $5 \times 5$ max filter to solve correspondence problem upto some extent. Then the scatter matrices $S_{1}$ and $S_{2}$ of dimension $256 \times 256$ are formed corresponding to $\left(g_{i-1}, g_{i}\right)$ and $\left(g_{i+1}, g_{i}\right)$ respectively. In order to obtain $S_{m}$, two vectors $S_{v_{1}}$ and $S_{v_{2}}$ corresponding to $S_{1}$ and $S_{2}$ are formed, where the elements in $S_{v_{i}}$ are the normalized sum of the values along the diagonal and its parallels in $S_{i}$. Finally, the Bhattacharya distance between $S_{v_{1}}$ and $S_{v_{2}}$ is taken as $S_{m}$.

\subsection{Detection and Classification}

Thus, 9 features $<a_{i}, b_{i}, E_{i}, a_{i_{p}}, e_{i_{p}}, b_{i_{n}}, e_{i_{n}}, I_{\text {avg }}, S_{m}>$ corresponding to each frame is obtained. As the model of equation 5 is being used in our frame estimation process, a classification scheme is required to detect and classify the shot boundaries. Here, we have relied on neural network based approach and a Multilayer Perceptron (MLP) network has been used. MLP network is a multi-class classifier consisting of several layers of neurons of which first one is the input layer and the last one is the output layer, remaining layers are called hidden layers. In the architecture employed here, there are complete connections between the nodes in successive layers but there is no connection between the nodes within a layer. Corresponding to each frame an input vector is provided to the network. Corresponding to $i$-th frame, the input feature vector is of 27-dimensions which is formed by putting together the features of $(i-1)$-th, $i$-th and $(i+1)$-th frames. Thus, the feature vector for a frame also relies on previous and following frames. During training phase, along with the input vector, a label denoting the class 
Table 1. Confusion Matrix and Accuracy for Training Data

\begin{tabular}{|c|c|c|c|c|}
\hline \multirow{2}{*}{$\begin{array}{c}\text { Actual } \\
\text { Class }\end{array}$} & \multicolumn{2}{|c|}{ Recognized Class } & Classification \\
Ac & gc & ac & Accuracy \\
\hline nc & 7051 & 112 & 0 & $98.44 \%$ \\
\hline gc & 141 & 537 & 0 & $79.2 \%$ \\
\hline ac & 0 & 0 & 57 & $100.0 \%$ \\
\hline
\end{tabular}

Table 2. Confusion Matrix and Accuracy for Test Data

\begin{tabular}{|c|c|c|c|c|}
\hline \multirow{2}{*}{$\begin{array}{c}\text { Actual } \\
\text { Class }\end{array}$} & \multicolumn{3}{|c|}{ Recognized Class } & Classification \\
\cline { 2 - 4 } & nc & gc & ac & Accuracy \\
\hline nc & 7028 & 133 & 2 & $98.12 \%$ \\
\hline gc & 160 & 517 & 0 & $76.37 \%$ \\
\hline ac & 1 & 0 & 56 & $98.25 \%$ \\
\hline
\end{tabular}

of the frame (i.e. no change, abrupt change or gradual change) is also provided. Then the connection weights are set such that the error between the network output and the target output (i.e. the classification error) becomes minimum.

\section{$3 \quad$ Experimental Results}

The frames are manually groundtruthed and the feature vectors are labeled accordingly. The frames are classified into three categories such as no change (nc), gradual change $(g c)$ and abrupt change (ac). The frames within a shot belong to $n c$. The successive frames where abrupt change (cut) occurs are marked as $a c$ and the frames under gradual transition (dissolve, fade-in, fade-out) are labeled as class $g c$. The dataset for this experiment consists of 15, 765 frames collected from various video files like BOR03, BOR19, UGS04, UGS09 etc. present in TRECVID 2001 test database downloaded from http://www.open-video.org. It contains different types transitions like abrupt change(cut), fade-in, fade-out and dissolve.

In our experiment, MLP network has only one hidden layer. Number of hidden nodes is chosen experimentally and set to 15 . It relies on back-propagation learning. The learning rate is 0.6 and the number of iteration used for training is 15,000 . The training dataset is generated by randomly choosing $50 \%$ frames of each category present in the dataset and the rest are used as the test dataset. The experiment is repeated several times by selecting different training and test dataset. The average result in the form of confusion matrix is shown in tables 1 and 2. The overall training and testing accuracy achieved are $96.79 \%$ and $96.25 \%$ respectively. Thus, it can be argued that the proposed methodology is capable enough to classify the frames reliably. As it was indicated, confusion occurs between gradual transitions (more specifically, the dissolve) and frames within the shot. It has occurred mostly because of the presence of camera motion, zooming effect, very slow paced gradual transitions etc. 


\section{Conclusion}

We have presented and adhered to a unified model of shot transitions. Considering the model as underlying framework, a scheme is proposed to identify a frame based on its previous and following frames. The transition parameters along with the scatter matrix of edge strength and average intensity describes a frame. For classification, we have employed a Neural Network with back-propagation. It classifies the frame into one of the three categories: no change, gradual change or abrupt change. Thus, a unified model based scheme is presented which is free from the critical issues like various threshold or window size selection.

\section{References}

1. Cabedo, X.U., Bhattacharjee, S.K.: Shot detection tools in digital video. In: Proc. Non-linear Model Based Image Analysis, pp. 121-126. Springer, Heidelberg (1998)

2. Zhang, H.J.: Automatic partitioning of full-motion video. ACM/Springer Multimedia Systems 1(1), 10-28 (1993)

3. Yeo, B., Liu, B.: Rapid scene analysis on compressed video. IEEE Trans. on Circuits and Systems for Video Technology 5(6), 533-544 (1995)

4. Patel, N.V., Sethi, I.K.: Video shot detection and characterization for video databases. Pattern Recognition 30(4), 583-592 (1997)

5. Huang, C.L., Liao, B.Y.: A robust scene-change detection method for video segmentation. IEEE Trans. on Circuits and Systems for Video Technology 11(12), 1281-1288 (2001)

6. Zabih, R., Miller, J., Mai, K.: A feature based algorithm for detecting and classifying scene breaks. In: Proc. ACM Multimedia 1995, pp. 189-200 (1995)

7. Hanjalic, A.: Shot-boundary detection: Unraveled and resolved? IEEE Trans. on Circuits and Systems for Video Technology 12(2), 99-104 (2002)

8. Cernekova, Z., Pitas, I., Nikou, C.: Information theory-based shot cut/fade detection and video summarization. IEEE Trans. on Circuits and Systems for Video Technology 16(1), 82-91 (2006)

9. Hampapur, A., Jain, R., Weymouth, T.: Production model based digital video segmentation. Multimedia Tools and Applications 1, 1-38 (2002)

10. Zabih, R., Miller, J., Mai, K.: A feature based algorithm for detecting and classifying production effects. Multimedia Systems 7(2), 119-128 (1999)

11. Lienhart, R.: Reliable dissolve detection. In: Proc. SPIE conf. on SRMD(4315), pp. 219 - 230 (1999)

12. Porter, S., Mirmehdi, M., Thomas, B.: Detection and classification of shot transitions. In: Proc. 12th British Machine Vision Conference, pp. 73-82. BMVA press (2001)

13. Yoo, H.W., Ryoo, H.J., Jang, D.S.: Gradual shot boundary detection using localized edge blocks. Multimedia Tools and Applications 28, 283-300 (2006) 